Slavica

bruxellensia

\section{Slavica bruxellensia}

Revue polyphonique de littérature, culture et histoire

slaves

$2 \mid 2009$

Femme(s)

\title{
Entretien avec Petra Hůlová
}

\section{Sarah Flock et Richard Vacula}

Traducteur : Sarah Flock

\section{(2) OpenEdition}

Journals

Édition électronique

URL : http://journals.openedition.org/slavica/171

DOI : 10.4000/slavica. 171

ISSN : 2034-6395

Éditeur

Université libre de Bruxelles - ULB

Édition imprimée

Pagination : 49-55

ISSN : 2031-7654

Référence électronique

Sarah Flock et Richard Vacula, "Entretien avec Petra Hůlová », Slavica bruxellensia [En ligne], 2 | 2009, mis en ligne le 09 mai 2012, consulté le 27 avril 2019. URL : http://journals.openedition.org/ slavica/171 ; DOl : 10.4000/slavica.171

Ce document a été généré automatiquement le 27 avril 2019

\section{(c) (i) (9)}

Les contenus de Slavica bruxellensia sont mis à disposition selon les termes de la Licence Creative Commons Attribution - Pas d'Utilisation Commerciale - Pas de Modification 3.0 France. 


\title{
Entretien avec Petra Hůlová
}

\author{
Sarah Flock et Richard Vacula \\ Traduction : Sarah Flock
}

\section{Présentation}

1 Petra Hůlová compte parmi les jeunes auteurs les plus importants de la scène littéraire contemporaine tchèque. Née le 12 juillet 1979 à Prague, elle a étudié la culturologie ${ }^{1}$ et la langue mongole à l'Université Charles de Prague. Elle est aujourd'hui docteur en culturologie. Son premier roman, PaměŤ mojí babičce, a paru aux éditions Torst en 2002. Il a été primé par le prix Magnesia Litera ${ }^{2}$ dans la catégorie découverte de l'année. Ce roman, également traduit en français, a été publié en 2005 aux éditions de l'olivier, sous le titre Les montagnes rouges ${ }^{3}$. Les éditions Torst, située à Prague, ont édité tous ses romans: en 2004 sort Přes matný sklo (À travers le verre dépoli); en 2005, Cirkus Les Mémoires (Le cirque Les Mémoires); en 2006, Umèlohmotný tř́ípokoj (Un trois pièces en toc), récompensé par le prix Jiří Orten ${ }^{4}$. En 2008, son cinquième roman, Stanice Tajga (La gare de Tajga) a paru et a reçu le prix Josef Škvoreckýs.

\section{Entretien}

\section{Débuts littéraires}

Richard Vacula: Votre premier livre est inspiré par la Mongolie qui a été l'objet de vos études et même le but de votre séjour d'études. La question s'impose donc: Dans quelle mesure ce pays a-t-il été le mécanisme déclencheur de votre parcours littéraire ? Quel rôle a-t-il joué dans votre décision de vous consacrer à l'écriture?

C'est difficile à dire. Je pense cependant que plus important encore que la Mongolie, c'est le froid, le mal du pays et énormément de temps libre qui ont été déterminants. Je n'ai pas commencé à écrire à cause du pays, mais parce que j'avais besoin de tuer le temps pendant mon stage en Mongolie. À ce moment-là, je n'allais pas très bien psychologiquement et j'avais besoin d'orienter mes pensées autrement qu'en les 
laissant tourner sur elles-mêmes. La Mongolie n'a pas vraiment amorcé mon écriture mais a plutôt été un épisode par lequel mon premier roman est devenu si populaire en République tchèque.

R. V. : Au cours de l'élaboration de ce livre, avez-vous eu des entretiens avec des Mongols ? Non, je n'ai pas eu d'entretiens.

R. V. : Avez-vous eu des échos des Montagnes rouges en Mongolie, qu'ils proviennent du cercle de vos amis ou d'ailleurs ? Évidemment sous la condition que votre livre ait été lu par des Mongols parlant tchèque.

Oui, j'en ai eu. La presse mongole a publié une critique négative. Elle désapprouvait qu'une étrangère s'autorise à écrire sur la prostitution et la pauvreté en Mongolie. Elle percevait le livre comme si je dénigrais la Mongolie. Toutefois, j'ai également eu quelques échos positifs de Mongols qui m'ont dit que ce serait magnifique si un Mongol écrivait un tel livre. Cela m'a beaucoup touchée.

R. V. : Comment combiner études et création littéraire? Est-il difficile de s'aménager des plages temporelles pour l'un et pour l'autre? Et comment vos collègues et professeurs perçoivent-ils ou ont-ils perçu votre activité littéraire?

Aujourd'hui, je n'étudie plus. En avril 2008, j'ai soutenu ma thèse en culturologie à la Faculté de Philosophie de l'Université Charles de Prague. Ce n'était pas difficile d'étudier et d'écrire en même temps, j'écrivais toujours à l'étranger ou pendant l'été, quand il n'y avait pas de cours.

\section{Écriture}

R. V. : La langue tchèque se caractérise par ses différents registres. II existe une langue parlée et une langue littéraire. Dans la majorité de vos romans, vous utilisez la langue non littéraire. Quel sens lui appliquez-vous dans votre œuvre?

Je n'arrive simplement pas à écrire autrement.Je n'ai pas choisi d'utiliser la langue parlée en écrivant. Elle s'est en quelque sorte imposée comme une nécessité et ne me définit pas du tout. En bref, j'ai des problèmes à écrire en langue littéraire, ça ne coule pas naturellement, ça ne va pas. Et même, ça m'énerve, j'aurais vraiment aimé écrire en utilisant la langue littéraire, mais ce n'est pas possible.

Sarah Flock: L'intrigue de vos romans prend place dans des lieux qui vous sont généralement familiers, soit par vos études, soit par vos nombreux voyages. Vous avez vécu à Oulan Bator et à New York, séjourné à Saint-Pétersbourg, en Sibérie... Quelle importance prend votre expérience personnelle au sein de vos récits? Je pense notamment au personnage de Tereza, votre premier personnage féminin de nationalité tchèque...

Mon expérience personnelle se reflète évidemment d'une certaine façon dans mes textes, mais je ne parlerais pas d'autobiographie directe.En ce qui concernepar exemple le personnage de Tereza, le personnage même est inspiré d'un photographe tchèque, un ami qui enseigne pour le moment la photographie à l'UMPRUM ${ }^{6}$ et qui a essayé de percer à New York. La rencontre entre Tereza et Ramid est, quant à elle, influencée par une rencontre que j'ai eue avec un homme qui s'appelait Rahim, et avec lequel j'ai sympathisé à New York. 
R. V. : Dans vos livres, vous touchez à la sincérité, à une exposition authentique des points de vue de vos différents personnages...

Je n'ai rien d'autre que moi-même et la possibilité d'imaginer ce que les autres personnes pensent et sentent. Évidemment, j'entends, vois et m'imagine aussi quelque chose.

S. F. : Il s'agit de l'empathie de l'auteur pour ses personnages. Pensez-vous que cette force empathique tienne de la forme privilégiée du monologue et de votre préférence pour le monologue intérieur?

J'ai toujours eu plus facile d'écrire des monologues et des monologues intérieurs. Les dialogues me semblent généralement plus difficiles. Il m'est quasiment impossible d'écrire une conversation ordinaire entre deux personnes et de la trouver convaincante. En revanche, je crois que je peux me représenter le monologue intérieur de différents individus compliqués et que je peux aussi l'écrire.

R. V. : Vos romans sont tour à tour situés dans des réalités étrangères puis tchèques. Estce que cette alternance est fortuite ou revêt-elle sa propre logique? Puisez-vous votre inspiration dans le changement du lieu géographique?

C'est certainement l'étranger qui m'inspire le plus. Il ne s'agit toutefois pas seulement de sa "couleur", il s'agit surtout du fait qu'à l'étranger, je perçois les choses de manière plus alerte et plus intense. D'ailleurs c'est toujours à l'étranger que sont nés tous mes livres, et ce depuis le premier. J'écris tout simplement mieux ailleurs.

R. V. : Pour vous, est-il plus exigeant d'écrire un roman dont l'intrigue se déroule dans un environnement tchèque ou étranger? Les deux types d'intrigue ont sans aucun doute leurs écueils...

Il est certainement plus exigeant d'écrire un roman portant sur la réalité tchèque. La description d'un environnement «exotique» est en soi intéressante, alors que la description d'un milieu tchèque ne l'est pas tellement, du moins c'est très difficile de la rendre intéressante, aussi la critique littéraire ose plus envers vous dans le cas d'un "roman tchèque ». Chaque Tchèque s'y connaît en Tchéquie, alors que dans le cas de la Mongolie, de la Sibérie ou des États-Unis, je peux dire à n'importe qui qu'il critique quelque chose dont il ne connaît pas grand-chose lui-même et, vraisemblablement, ce sera la vérité...

S. F. : Vous vous êtes frottée à l'art de la prose avec succès. Avec la complicité de Viktorie Čermáková7, votre roman Umělohmotný třípokoja été adapté pour le théâtre sous le titre de Česká pornografie (La pornographie tchèque). Quelle a été votre implication au sein de ce projet ? Quelle expérience en avez-vous retirée ?

$\mathrm{Au}$ début, je n'ai pas du tout cru à l'adaptation théâtrale de mon roman. Je me demandais comment transposer un monologue littéraire au théâtre.... Toutefois Viktorie Čermáková, qui a aménagé mon texte et l'a ensuite mis en scène, s'en est tirée à merveille. Mon rôle dans ce projet a été minimal, mais je me suis énormément amusée pendant la période des répétitions et j'ai beaucoup aimé voir comment un texte prend corps grâce à des personnes vivantes. J'ai parfois relevé ou expliqué quelque chose plus en profondeur, mais j'ai surtout profité de ma position de spectateur. Je suis fascinée par le théâtre.

\section{Au-delà de l'écriture}

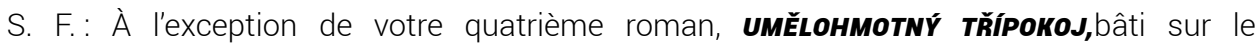
monologue d'un seul personnage, vos récits obéissent à une logique polyphonique qui 
entrecroise différents destins liés entre eux ou non. De même, vous vous amusez à combiner les espaces, par exemple la ville et la campagne pour Les Montagnes rouges; l'Asie, l'Europe et les États-Unis pour Cirkus Les Mémoires, et la temporalité: la Prague communiste et contemporaine dans Pres matný sklo, la fin des années 1940 jusqu'à nos jours en Sibérie pour votre roman Stanice Tajga ... Ce procédé vous amène à brosser le portrait psychologique de vos personnages sous un fond de fresque historique. $Y$ aurait-t-il chez vous une volonté de toucher à l'universel?

Je ne sais trop quoi répondre. Les récits et les personnes ont sans doute dans une certaine mesure un caractère universel. Il existe plusieurs types de personnes, et plusieurs types de récits. Toutefois, cela n'élimine pas l'absence de limites des variantes des deux...

S. F. : Dans l'enchevêtrement des vies de vos personnages, on peut observer une répétition inconsciente et involontaire du schéma familial : similarité entre le parcours de Dzaja et celui de sa fille, continuité entre les destins de Charil et de Ramid. Est-ce une croyance, une hypothèse qui vous est propre? Pensez-vous que les modèles familiaux, par extension sociaux et historiques, se répètent?

C'est une question intéressante, personne ne me l'avait encore posée et moi-même je ne m'étais pas non plus aperçue du parallèle des liens entre Charil et Ramid ou Dzaja et Dolgorma. Mais vous avez raison, cette similitude est bel et bien là. Je m'intéresse assurément aux rapports des jeunes face aux personnes âgées. Et aussi, de la tradition face au dégoût qu'elle peut inspirer. Moi-même, dans ma vie personnelle, je me démène sans cesse dans quelque chose de similaire.

R. V. : Dans votre œuvre, vous abordez souvent la question du vieillessement, ou plutôt comment l'individu, et en particulier la femme, y fait face. Êtes-vous personnellement effrayée par la vieillesse et le vieillissement en tant que processus ? L'écriture représente-telle pour vous un des moyens de se préserver de la fuite du temps?

La fuite du temps est peut-être ce qui m'intéresse le plus. Je n'ai pas peur de la vieillesse, mais elle me fascine, c'est vrai. Je pense que, tant qu'un homme n'est pas âgé, il ne sait pas ce que c'est. Ce qui m'effraie c'est l'incommunicabilité de cette expérience. Je m’intéresse aussi aux différents tabous qui sont liés à la veillesse.

\section{Thèmes}

S. F. : Votre premier roman, Les Montagnes rouges, est écrit sous le sceau de la féminité. Une écrivaine met en scène les chants de cinq femmes différentes. Au sein de votre écriture, les caractères féminins sont multiples. Toutefois, on peut observer une récurrence dans certains motifs, et ce au fil de vos romans. Par exemple, la dimension chamanique ou, dans une moindre mesure la propension à l'ésotérisme qui apparaît chez les personnages, chez les femmes asiatiques d'un certain âge, tels que Chiriko dans Les Montagnes rouges, ou Sula et Taura dans Cirkus - Les Mémoires. La mère d'Ondřej que l'on retrouve dans Přes matný sklo est, quant à elle, animée par un désir de spiritualité...

La féminité en tant qu'aptitude à manier une certaine magie et à accéder aux choses irrationnelles était quelque chose qui m'intéressait et en quoi je croyais d'une certaine façon.Maintenant, je le vois un peu différemment, comme une sorte de conception romantique. Les personnages de vieilles sorcières m'ont beaucoup intéressée et je pense qu'elles m'ont suivie sur le papier. Néanmoins, j'ai fait le tour de ce thème aujourd'hui.

S. F.: Par votre œuvre, vous multipliez les points de vue sur un même sujet. Ainsi la prostitution se trouve déclinée sous plusieurs formes dans vos romans. Elle est traitée de manière édulcorée dans Les Montagnes rouges (je pense aux personnages deDzaja, 
Dolgorma junior, Nara et Chartsetseg) évoquée par Amira et Nila dans Cirkus Les mémoires et décrite sous une plume très crue dans Umělohmotný třípokoj.

Vous avez raison en remarquant que ce thème se répète. Et ça se passe sans que j'en sois vraiment consciente, sans que je ne le recherche. Simplement, ce thème revient. Je ne sais pas moi-même pourquoi. Il y a comme ça beaucoup de choses que je ne sais pas, que je ne parviens pas à m'expliquer sur mon écriture. Voire à peu près tout.

S. F. : La bâtardise perce également en filigrane au travers vos romans. Souvent il s'agit d'une bâtardise tabouisée, non reconnue. Je pense plus particulièrement aux personnages de Dzaja, de Dolgmora junior et de Charil, qui ne connaissent pas leur vrai père ; à l'enfant de Nara, qui ne connaît ni son père, ni sa mère...

Je pense que cela doit être influencé par le fait que j'ai moi-même des frères et sœurs illégitimes et que j'ai été élevée par deux pères. Les liens familiaux dans notre famille sont compliqués et cela se reflète nécessairement d'une certaine façon dans mon écriture.

S. F. : La majorité de vos personnages, peut-être tous, sont dans un état d'attente ou de recherche. Ce sont des exilés, des artistes ambulants, des aventuriers, des personnes ordinaires, qui rêvent d'une vie comme au cinéma. Ils sont assis à une table et ils regardent par la fenêtre en espérant qu'arrive l'objet de leur attente. Ce sont principalement des personnes désenchantées et seules...

Ils sont ainsi. J'attends moi-même aussi dans la majorité des cas.Je ne sais pas quoi, mais je reste dans cet état d'attente qui prend évidemment beaucoup d'importance dans ma vie. Je connais donc très intimement cet état et j'arrive très bien à me représenter ces personnages qui attendent, puis à les décrire. C'est sans doute pourquoi autant d'entre eux attendent... (rires). Et l'attente est également une occasion idéale pour le monologue intérieur que j'aime tant.

S. F.: Peut-être pourriez-vous renseigner nos lecteurs... Savez-vous si une nouvelle traduction française d'un de vos romans est prévue? Et si oui, savez-vous quand elle paraîtra?

La traduction en français deCirkus Les Mémoires est terminée. Mais il y a quelques jours, j'ai appris que la maison d'édition qui devait la publier venait de fermer. Autrement dit, la date de publication est reportée et mon agent essaie de trouver un nouvel éditeur...

R. V. : Avant de nous quitter, pouvez-vous nous confier quelles idées littéraires avez-vous actuellement en tête. Travaillez-vous sur un nouveau livre?

Pour l'instant, je ne travaille sur aucun livre. J'ai quelques idées, on verra bien.

S. F. \& R. V. : Au nom de SLAVICA BRUXELLENSIA, nous vous remercions pour cette agréable interview.

C'est moi qui vous remercie. J'ai répondu avec plaisir à vos questions. Bonjour à vos lecteurs. 


\section{NOTES}

1. Les études de culturologie n'existent pas en Belgique. À l'Université Charles, le cursus se consacre aux matières telles que la psychologie, l'ethnologie, la sociologie, l'archéologie, l'anthropologie ou encore la philosophie, et les aborde à travers le prisme des cultures.

2. Le prix Magnesia Litera promeut annuellement les meilleurs livres publiés l'année précédente. Il récompense huit catégories littéraires et attribue un prix du lecteur. Le jury est constitué par des membres de l'Académie des Sciences de la République tchèque, le centre tchèque de l'association P.E.N., les associations des écrivains, des traducteurs, des éditeurs et des libraires...

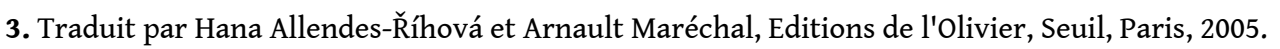

4. Le prix littéraire Jiří Orten, du nom du jeune écrivain tchèque mort prématurément, récompense chaque année un auteur tchèque de moins de trente ans. Ce prix existe depuis 1982 et a été notamment attribué à Michal Vieweg en 1993.

5. Le prix Josef Škvorecký a été décerné pour la première fois en 2007 à l'auteur Jan Novák. Mue par la volonté de ne plus récompenser uniquement ses élèves, l'académie littéraire (la haute école privée Josef Škvorecký) attribue désormais un prix à un auteur tchèque dont le roman a paru dans le courant de l'année écoulée ; en cas d'égalité, la voix de l'auteur tranche.

6. Ecole supérieure des Arts et Métiers à Prague.

7. Viktorie Čermáková (1966, Prague) est comédienne et metteur en scène, jeune diplômée de la Damu. Elle débute en tant que comédienne amateur et rejoint vite des théâtres importants à l'instar du Divadlo Husa na provázku (Théâtre l'Oie en laisse) à Brno ou Divadlo Komedie (Théâtre Comédie) à Prague. Elle joue notamment sous la direction de Michal Dočekal, Jan Nebeský, Jiřív Pokorný ou encore de Jan Antoním Pitínský. En 2006, elle co-fonde le studio théâtre l'Usine, Divadelní studio Továrna qui a l'habitude de collaborer avec le lieu alternatif La Fabrika (la Fabrique). En 2007, elle a été récompensée lors du festival Next Wave pour son apport à la scène tchèque. La même année, elle a également été nominée pour le prix Alfréd Radok.

\section{INDEX}

Index chronologique : XXe siècle

Mots-clés : littérature tchèque

Index géographique : République tchèque

\section{AUTEURS}

\section{SARAH FLOCK}

Aspirante du FNRS à l'Université Libre de Bruxelles (Belgique) et à l'Université Charles de Prague (République tchèque) 


\section{RICHARD VACULA}

Lecteur de langue tchèque à l'Université Libre de Bruxelles (Belgique) 